\title{
Optimising islet engraftment is critical for successful clinical islet transplantation
}

\author{
O. Korsgren • T. Lundgren • M. Felldin • A. Foss • \\ B. Isaksson • J. Permert • N. H. Persson • E. Rafael • \\ M. Rydén • K. Salmela • A. Tibell • G. Tufveson • \\ B. Nilsson
}

Received: 27 June 2007 / Accepted: 17 September 2007 / Published online: 27 November 2007

(C) Springer-Verlag 2007

\begin{abstract}
Clinical islet transplantation is currently being explored as a treatment for persons with type 1 diabetes and hypoglycaemia unawareness. Although 'proof-of-principle' has been established in recent clinical studies, the procedure suffers from low efficacy. At the time of transplantation, the isolated islets are allowed to embolise the liver after injection in the portal vein, a procedure that is unique in the area of transplantation. A novel view on the engraftment of intraportally transplanted islets is presented that could explain the low efficacy of the procedure.
\end{abstract}

Keywords Clinical · Engraftment · Innate immunity · Intraportal $\cdot$ Intramuscular $\cdot$ Islet transplantation ·

\footnotetext{
O. Korsgren $(\triangle) \cdot$ B. Nilsson

Department of Radiology, Oncology and Clinical Immunology, Division of Clinical Immunology, Rudbeck Laboratory,

C11, University Hospital,

SE 75185 Uppsala, Sweden

e-mail: olle.korsgren@klinimm.uu.se

T. Lundgren $\cdot$ A. Tibell

Department of Transplantation Surgery, CLINTEC,

Karolinska Institute,

Stockholm, Sweden

M. Felldin

Department of Transplantation, University Hospital, Gothenburg, Sweden
}

\section{A. Foss}

Department of Transplantation Surgery,

Rikshospitalet-Radiumhospitalet Medical Center,

Oslo, Norway

B. Isaksson · J. Permert • E. Rafael

Department of Surgery, Karolinska Institute,

Stockholm, Sweden
Liver morphology · Thrombosis · Tumour metastasis .

Revascularisation

Clinical islet transplantation is currently being explored as a treatment for patients with type 1 diabetes who experience recurrent severe hypoglycaemic episodes. The procedure is highly successful, with $>80 \%$ of the recipients being protected from hypoglycaemic episodes for as much as 5 years after transplantation; most of the recipients then have to resume insulin therapy, but at lower doses than originally [1]. The insulin secretion capacity in these recipients has been estimated to be only $\sim 20-40 \%$ of that in a healthy person $[2,3]$, even when islets are obtained

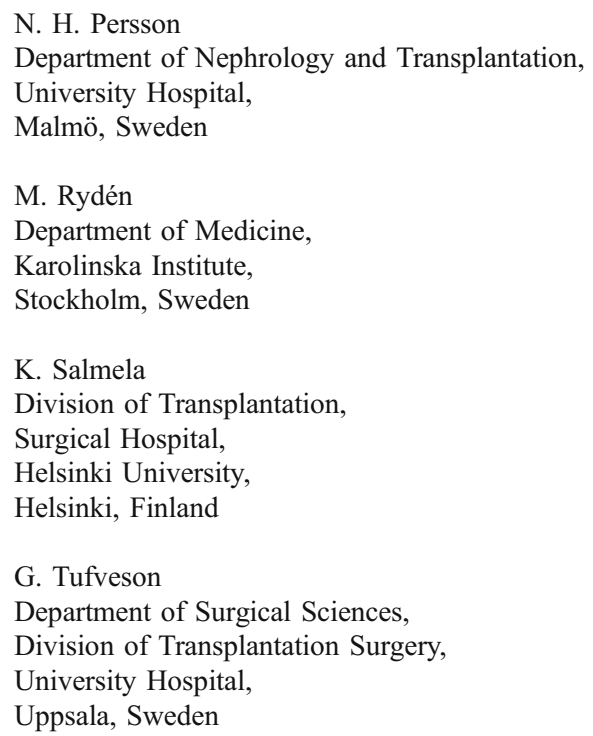


from two to four donors $[1,4]$, or in some studies as many as eight to 12 donors $[5,6]$.

The survival of islets from organ procurement to islet isolation and transplantation is depicted in Fig. 1. As in the case of all transplanted organs, there seems to be a gradual loss of islet function over time [1]. Even a minor reduction in beta cell mass in a patient not receiving insulin but with only $20-40 \%$ of normal islet mass would lead to the patient requiring exogenous insulin therapy at a low dose. This reduction in endocrine function may depend on the side effects of immunosuppression [7,8], but is likely to be reinforced by phenomena such as glucose [9] and lipid toxicity $[10,11]$.

\section{Engraftment of cells and islets embolised in the portal vein}

At the time of transplantation, the isolated islets are allowed to embolise the liver after infusion into the portal vein, a procedure that is unique in the area of organ transplanta-

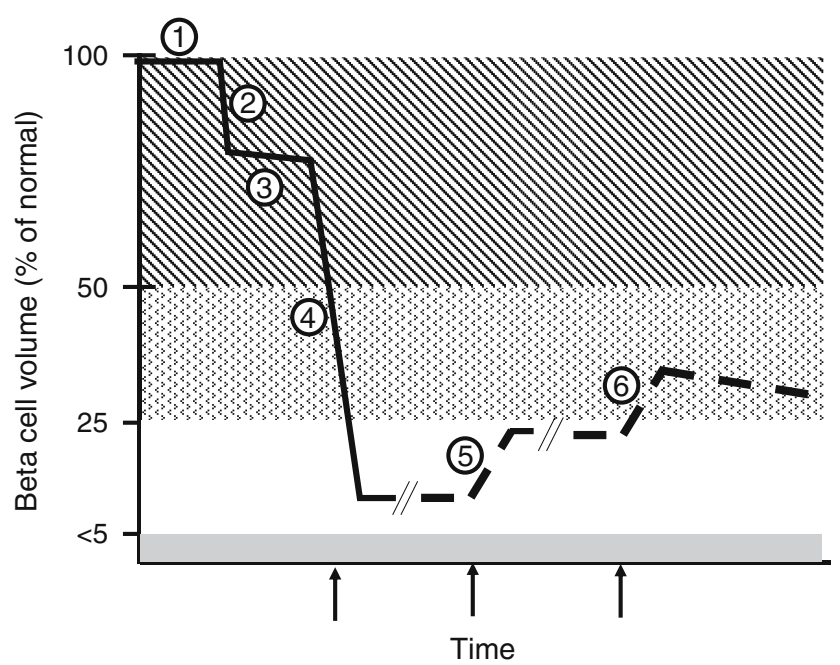

Fig. 1 Depiction of the beta cell mass after clinical islet transplantation. The normal beta cell mass in humans is considered to be $100 \%$; if it is reduced to $<50 \%$, most patients develop signs of impairment in glucose metabolism (e.g. postprandial hyperglycaemia). If further reduced to $<25 \%$, exogenous insulin therapy is required. The beta cell mass in a person with long-standing type 1 diabetes is usually $1-5 \%$ of that in a healthy individual. Against this background, the tentative islet mass in a donor is illustrated. (1) The islet mass in a donor is assumed to be close to $100 \%$, although the quality of the islets may be influenced by the cause of death and the process of brain death. (2) About $60-80 \%$ of the islets can be successfully retrieved in an experienced islet isolation facility. (3) About $10 \%$ of the islets are lost during culture while awaiting transplantation. (4) Most islets fail to engraft after intraportal islet transplantation. The estimated islet replacement level is about $10 \%$ after each transplantation procedure. (5) If the procedure is repeated (dotted line) once or twice (arrows) (6) the total beta cell mass reaches a level at which insulin can be withdrawn [2, 3], although most recipients show impaired glucose metabolism (postprandial hyperglycaemia) tion. Tumours in the gastrointestinal tract are common, and a feared complication is metastasis to the liver, i.e. a situation similar to islet transplantation. This process is multi-step [12], involving: (1) growth of the primary tumour to the extent that a local venous blood vessel is penetrated; (2) seeding of tumour cells into the bloodstream; (3) intraportal entrapment of the tumour cells due to size restraints; (4) adherence of the tumour cells to the vessel wall; (5) penetration through the vessel wall; and finally (6) growth and initiation of the process of revascularisation within the parenchyma of the liver [13]. Only if all these steps are successfully completed can metastasis be established. The efficacy of the engraftment of tumour cells seems to be extremely low. In a rat model of colon cancer metastasis entailing the injection of $5 \times 10^{6}$ tumour aggregates into the portal vein, only few metastases were established; when $0.5 \times 10^{6}$ tumour aggregates were injected, no metastases were formed; and when $10^{7}$ cell aggregates were injected, three out of five rats died as a result of portal thrombosis [14].

Islets injected into the portal vein should follow the same steps to successfully complete the engraftment process. However, once they have become entrapped in the portal vein because of size restrictions, the isolated islets have only a limited capacity to actively adhere to and penetrate the venular wall.

Entrapment of islets as a result of size restriction in the portal veins may cause a drop in pressure in the portal vein distal to the islet, a change that could markedly alter the regional blood flow. The portal vein and the hepatic artery follow the same branching system throughout their route within the liver (Fig. 2a-c). Both vessels branch repeatedly and empty into the sinusoids (capillaries) of the liver parenchyma. The low pressure formed in the portal vein distal to an entrapped islet could cause a back-flow of blood from small arterial branches. The blood encompassing the islet will subsequently be delivered via portal vein branches into the surrounding sinusoids.

\section{Revascularisation of intraportally transplanted islets}

To our knowledge, there are no reports of one vessel growing into the lumen of another vessel. The basement membrane of the endothelial cell lining seems to constitute an insurmountable biological barrier to other vessels. Also, the flow of blood around the entrapped islets prevents the establishment of physiological concentration gradients of relevant growth factors (e.g. fibroblast growth factor [FGF] and vascular endothelial growth factor A [VEGFA]), which are released from the transplanted islets, across the blood vessel wall to stimulate the penetration of new blood vessels into an existing vessel. A single standardised islet 

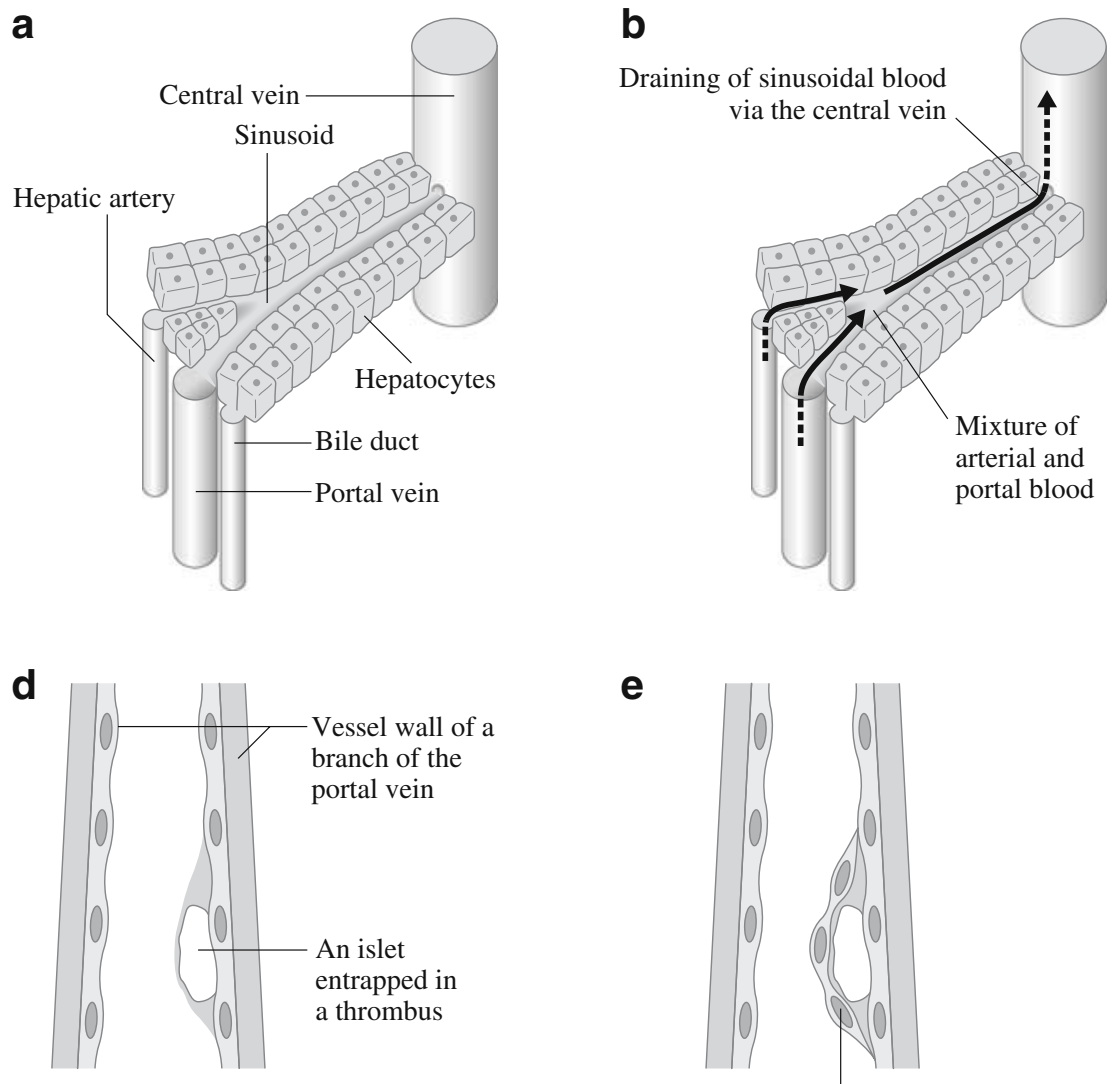

e
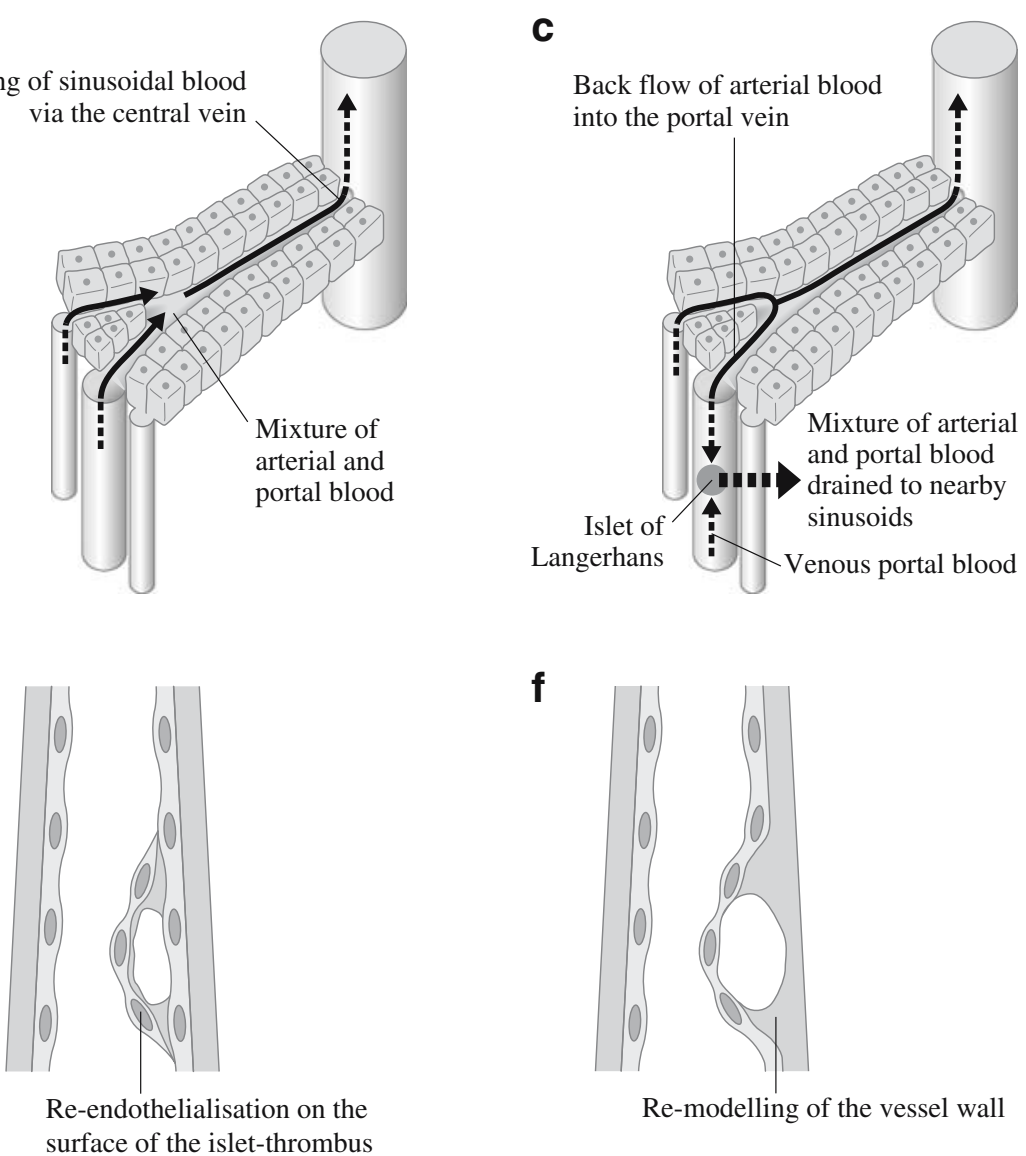

f

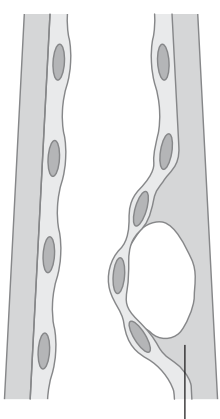

Re-modelling of the vessel wall
Fig. 2 A model of islet revascularisation after intraportal transplantation. a Normal liver anatomy. b Normal blood perfusion of the liver. c Islets entrapped as a result of size restriction in the portal vein will induce low pressure in the vessel distal to the islet. This low pressure distal to the entrapped islet could cause a back-flow of arterial blood into the portal vein. The blood encompassing the islet will subsequently be delivered via portal vein branches into the surround- ing sinusoids. d An islet-thrombus entrapped in a portal branch adheres to the vessel wall and is over time partly resorbed. e Reendothelialisation occurs on the surface of the clot to allow recanalisation of the vessel lumen. $\mathbf{f}$ The vessel wall is re-modelled. During this process the islet becomes incorporated in the vessel wall and may eventually receive blood supply from the vas vasorum equivalent (IEQ), with an average diameter of $150 \mu \mathrm{m}$, generally becomes entrapped in a relatively large branch of the portal vein. These branches have a complete vessel wall with its various layers, making it even more unlikely that a proper revascularisation process will be induced. In line with this notion is a recent study from a group in Zurich showing superior functional outcome of small islets compared with large islets in clinical transplantation [15].

The relative success of intraportal islet transplantation in rodents is not consistent with the scenario described above, probably because of the large size of rodent islets compared with the diameter of the portal veins in these animals. Rodent islets are often reported to be even larger than human islets, and if injected in the portal vein they generate large necrotic areas (Fig. 3) due to acute ischemia induced by the sudden obstruction of a large portal branch. This process initiates a cascade of growth factors that stimulate hepatocyte proliferation and re-modulation of the liver parenchyma. Several noteworthy studies in rodents have

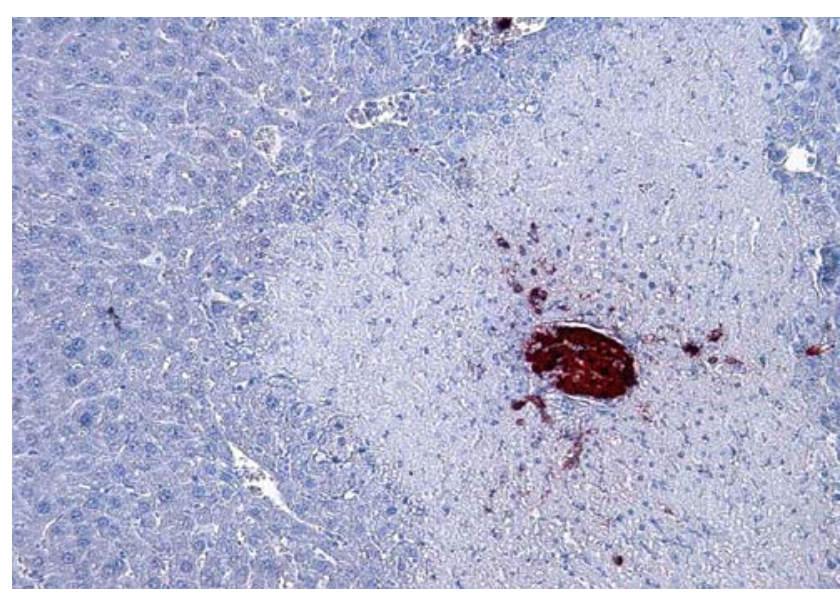

Fig. 3 Experimental intraportal islet allotransplantation in mice. An islet (dark brown; immunostained for insulin) entrapped by size restriction in a branch of the portal vein day 1 after transplantation. A large hepatic necrotic area has developed in the surrounding hepatic parenchyma 
demonstrated that transplanted islets respond with proliferation and improved engraftment when exposed to growth factors released during liver regeneration [16, 17]. By this process, the islets become incorporated in the newly formed liver parenchyma and induce revascularisation from the hepatic artery $[18,19]$ within the first week after transplantation (cf. tumour cells actively penetrating the portal venous wall).

\section{Observations in human and non-human primates}

In humans $[20,21]$ or non-human primates [22-30], islets entrapped in the portal veins after intraportal transplantation seem unable to escape the vascular compartment. In fact, we have been unable to find any publication reporting the presence, in non-human primates or humans, of intraportally transplanted islets located in the liver parenchyma. Instead, all the published studies show the islets within the portal vein lumen or incorporated in portal vein walls, several months or even years after transplantation. Also, in humans or non-human primates most islets are recognised by the innate immune response and become covered with fibrin and platelets (i.e. the instant blood-mediated inflammatory reaction [IBMIR] occurs [31]) (Fig. 2d-f). If an islet escapes the other detrimental features of the IBMIR, these platelets may actually facilitate adherence to the vessel wall. The resulting islet-thrombus, like all clots, is at least partially resolved over time, leaving fibrin strands extending between the vessel wall and the islet. Finally, endothelial cell coverage demarcates the islet-thrombus mass from the vessel lumen, allowing the re-canalisation of the lumen of the portal vein, with the blood being able to bypass the site of islet adherence. Through this process, the islet finally becomes incorporated into the vessel wall. It is possible that such islets will become revascularised and receive blood from the vas vasorum. However, this process is slow and cannot be initiated until the original vessel wall has been re-modelled.

\section{Revisiting the rationale for selecting the intraportal route of administration for clinical islet transplantation}

The late P. Lacy and his co-authors, working with a rat model of diabetes, concluded that the liver was the optimal site for islet transplantation [32]. However, the other sites evaluated in his comparative study were (1) the intraperitoneal injection of free islets or (2) their subcutaneous transplantation, neither of which support islet survival. Other studies in rodents have clearly shown that transplantation under the kidney capsule produces superior longterm results compared with intraportal transplantation [33], and results in dogs have shown superior long-term outcomes after intrasplenic compared with intraportal islet transplantation $[34,35]$. The argument that insulin would be delivered more physiologically after intraportal transplantation has received little support in the literature. It has clearly been demonstrated in experimental studies that intraportally transplanted islets respond to glucose stimulation only when perfused via the hepatic artery; no response is observed after challenge via the portal vein [19]. Similar findings come from the area of tumour biology (cf. delivery of anti-cancer drugs or for interventions designed to occlude the blood supply to the tumour).

Disturbing results have come from a large and wellconducted study of diabetic BB rats receiving intraportal islet transplants (without the need for streptozotocin to induce diabetes or for any immunosuppressive drugs) [36]: in rats with long-term partial function, there was a remarkably high incidence of adenomas, and even hepatocellular carcinoma. The initial stages in the process leading to these pathological conditions were hepatocyte steatosis and glycogen deposits in the vicinity of the transplanted islets, findings that are analogous to those reported in patients from several transplant centres [37, 38]. In addition, islets in the liver are exposed to toxic products from the gastrointestinal tract, including high concentrations of the immunosuppressive drugs being administered $[7,8]$.

\section{Alternative sites for clinical islet transplantation}

An obvious solution to the problems discussed above would be to establish an alternative site for clinical islet transplantation. One of the most attractive sites that has been proposed thus far is the omental pouch [39], into which islets can be transplanted via a laparoscopic procedure. Several groups are already working to address this challenge in non-human primates ([40]; J. Contreras, University of Alabama at Birmingham, personal communication; B. Hering, University of Minnesota, personal communication). An advantage to this route is that insulin would be delivered to the liver via the portal vein. The details of the procedure are currently being explored in several ongoing studies in non-human primates.

A readily available alternative would be to use the same site for islet transplantation that has been employed successfully for decades in the autotransplantation of parathyroid glands [41]. The parathyroid glands are small multiple organs composed of highly metabolic active endocrine cells that produce parathormone, the only hormone that increases the concentration of extracellular $\mathrm{Ca}^{2+}$ in the body. These glands are highly vascularised and continuously sense the $\mathrm{Ca}^{2+}$ concentration in the blood; the 
release of parathormone is a direct response to changes in $\mathrm{Ca}^{2+}$ concentration. Since the half-life of parathormone in the circulation is about $5 \mathrm{~min}$, there is a need for a stringent and continuous $\mathrm{Ca}^{2+}$-sensing mechanism tied to a stimuluscoupled release of parathormone. The close relationship between the parathyroid glands and the islets is further illustrated by the fact that both organs are affected in the genetic disorder multiple endocrine neoplasia type 1 (MEN 1).

In parathyroid autotransplantation the glands are cut into small pieces and usually implanted between the muscle fibres in the forearm. Once in place, the endocrine cells begin a process of engraftment and revascularisation. The long-term outcome of the procedure is excellent, with almost no side effects [42]. The similarities between the parathyroid glands and pancreatic islets, as well as a few experimental [43, 44] and clinical observations [45-47] suggest that the intramuscular site holds promise for clinical islet transplantation also. If anything, the small size of the islets would facilitate their engraftment. The intramuscular site would also allow transplantation of composite isletendothelial cell grafts $[46,48]$ or the application of various matrices carrying growth factors that would facilitate the formation of a new endocrine organ at the new site of implantation. Indeed, in autotransplants, we have successfully transplanted isolated islets of Langerhans intramuscularly into the forearm of a patient undergoing total pancreatectomy because of recurrent life-threatening pancreatitis [49].

In conclusion, recent advances in biotechnology and cell therapy allow creation of an extrapancreatic insulin-producing organ, allowing transplantation to a site outside the liver, e.g. into the omental pouch or intramuscularly. The goal is to establish an extrapancreatic insulin-producing organ that initially is composed of islets, supporting cells and resorbable biomaterials to trigger high-density revascularisation of the islets and a complete integration into surrounding tissues. Several transplant centres are actively working to establish an alternative site for clinical islet transplantation as a first step to make this development possible. Procedures developed will be of immense importance not only for clinical islet transplantation but also for future stem cell therapy.

Acknowledgements This study was supported by grants from the Swedish Research Council, the Nordic Insulin Fund, the Ernfors Family Fund, Barn Diabetes Fonden, the Swedish Diabetes Association, the Juvenile Diabetes Foundation International and the National Institutes of Health. The authors would like to thank Vibggor HB and D. Woodroffe for the illustrations.

Duality of interest The authors declare that there is no duality of interest associated with this manuscript.

\section{References}

1. Shapiro AM, Ricordi C, Hering BJ et al (2006) International trial of the Edmonton protocol for islet transplantation. N Engl J Med $355: 1318-1330$

2. Keymeulen B, Gillard P, Mathieu C et al (2006) Correlation between beta cell mass and glycemic control in type 1 diabetic recipients of islet cell graft. Proc Natl Acad Sci USA 103:17444-17449

3. Ryan EA, Paty BW, Senior PA et al (2005) Five-year follow-up after clinical islet transplantation. Diabetes 54:2060-2069

4. Shapiro AM, Lakey JR, Ryan EA et al (2000) Islet transplantation in seven patients with type 1 diabetes mellitus using a glucocorticoidfree immunosuppressive regimen. N Engl J Med 343:230-238

5. Keymeulen B, Ling Z, Gorus FK et al (1998) Implantation of standardized beta-cell grafts in a liver segment of IDDM patients: graft and recipients characteristics in two cases of insulinindependence under maintenance immunosuppression for prior kidney graft. Diabetologia 41:452-459

6. van Kampen CA, van de Linde P, Duinkerken G et al (2005) Alloreactivity against repeated HLA mismatches of sequential islet grafts transplanted in non-uremic type 1 diabetes patients. Transplantation 80:118-126

7. Desai NM, Goss JA, Deng S et al (2003) Elevated portal vein drug levels of sirolimus and tacrolimus in islet transplant recipients: local immunosuppression or islet toxicity. Transplantation 76:1623-1625

8. Shapiro AM, Gallant HL, Hao EG et al (2005) The portal immunosuppressive storm: relevance to islet transplantation. Ther Drug Monit 27:35-37

9. Unger RH (1991) Diabetic hyperglycemia: link to impaired glucose transport in pancreatic beta cells. Science 251:1200-1205

10. Unger RH, Orci L (2002) Lipoapoptosis: its mechanism and its diseases. Biochim Biophys Acta 1585:202-212

11. Lee Y, Ravazzola M, Park B, Bashmakov Y, Orci L, Unger R (2007) Metabolic mechanisms of failure of intraportally transplanted pancreatic beta-cells in rats: role of lipotoxicity and prevention by leptin. Diabetes 56:2295-2301

12. MacDonald IC, Groom AC, Chambers AF (2002) Cancer spread and micrometastasis development: quantitative approaches for in vivo models. Bioessays 24:885-893

13. Gonda T, Ishida H, Yoshinaga K, Sugihara K (2000) Microvasculature of small liver metastases in rats. J Surg Res 94:43-48

14. Graf W, Sundin A, Glimelius B, Ahlstrom H, Carlsson J (1992) Induction and quantification of hepatic metastases from a human colonic cancer in the nude rat. Eur J Surg Oncol 18:608-614

15. Lehmann R, Zuellig RA, Kugelmeier P et al (2007) Superiority of small islets in human islet transplantation. Diabetes 56:594-603

16. Dunger A, Korsgren O, Andersson A (1990) DNA replication in mouse pancreatic islets transplanted subcapsularly into the kidney or intraportally into the liver. Influence of unilateral nephrectomy or partial hepatectomy. Transplantation 49:686-689

17. Nakano M, Yasunami Y, Maki T et al (2000) Hepatocyte growth factor is essential for amelioration of hyperglycemia in streptozotocin-induced diabetic mice receiving a marginal mass of intrahepatic islet grafts. Transplantation 69:214-221

18. Andersson A, Korsgren O, Jansson L (1989) Intraportally transplanted pancreatic islets revascularized from hepatic arterial system. Diabetes 1(38 Suppl):192-195

19. Lau J, Jansson L, Carlsson PO (2006) Islets transplanted intraportally into the liver are stimulated to insulin and glucagon release exclusively through the hepatic artery. Am J Transplant 6:967-975

20. Davalli AM, Maffi P, Socci C et al (2000) Insights from a successful case of intrahepatic islet transplantation into a type 1 diabetic patient. J Clin Endocrinol Metab 85:3847-3852 
21. Oberholzer J, Mathe Z, Bucher P et al (2003) Islet autotransplantation after left pancreatectomy for non-enucleable insulinoma. Am J Transplant 3:1302-1307

22. Adams AB, Shirasugi N, Durham MM et al (2002) Calcineurin inhibitor-free CD28 blockade-based protocol protects allogeneic islets in nonhuman primates. Diabetes 51:265-270

23. Adams AB, Shirasugi N, Jones TR et al (2005) Development of a chimeric anti-CD40 monoclonal antibody that synergizes with LEA29Y to prolong islet allograft survival. J Immunol 174:542550

24. Hirshberg B, Mog S, Patterson N, Leconte J, Harlan DM (2002) Histopathological study of intrahepatic islets transplanted in the nonhuman primate model using Edmonton protocol immunosuppression. J Clin Endocrinol Metab 87:5424-5429

25. Hirshberg B, Montgomery S, Wysoki MG et al (2002) Pancreatic islet transplantation using the nonhuman primate (rhesus) model predicts that the portal vein is superior to the celiac artery as the islet infusion site. Diabetes 51:2135-2140

26. Kawai T, Sogawa H, Koulmanda M et al (2001) Long-term islet allograft function in the absence of chronic immunosuppression: a case report of a nonhuman primate previously made tolerant to a renal allograft from the same donor. Transplantation 72:351-354

27. Kenyon NS, Fernandez LA, Lehmann R et al (1999) Long-term survival and function of intrahepatic islet allografts in baboons treated with humanized anti-CD154. Diabetes 48:1473-1481

28. Koulmanda M, Smith RN, Qipo A, Weir G, Auchincloss H, Strom TB (2006) Prolonged survival of allogeneic islets in cynomolgus monkeys after short-term anti-CD154-based therapy: nonimmunologic graft failure. Am J Transplant 6:687-696

29. Levisetti MG, Padrid PA, Szot GL et al (1997) Immunosuppressive effects of human CTLA4Ig in a non-human primate model of allogeneic pancreatic islet transplantation. J Immunol 159:51875191

30. Wijkstrom M, Kenyon NS, Kirchhof N et al (2004) Islet allograft survival in nonhuman primates immunosuppressed with basiliximab, RAD, and FTY720. Transplantation 77:827-835

31. Moberg L, Johansson H, Lukinius A et al (2002) Production of tissue factor by pancreatic islet cells as a trigger of detrimental thrombotic reactions in clinical islet transplantation. Lancet 360:2039-2045

32. Kemp CB, Knight MJ, Scharp DW, Ballinger WF, Lacy PE (1973) Effect of transplantation site on the results of pancreatic islet isografts in diabetic rats. Diabetologia 9:486-491

33. Hiller WF, Klempnauer J, Luck R, Steiniger B (1991) Progressive deterioration of endocrine function after intraportal but not kidney subcapsular rat islet transplantation. Diabetes 40:134-140

34. Evans MG, Warnock GL, Rajotte RV (1989) Comparison of sites for transplantation of canine pancreatic microfragments. Diabetes Res 10:35-41
35. Warnock GL, Dabbs KD, Evans MG, Cattral MS, Kneteman NM, Rajotte RV (1990) Critical mass of islets that function after implantation in a large mammalian. Horm Metab Res Suppl $25: 156-161$

36. Dombrowski F, Mathieu C, Evert M (2006) Hepatocellular neoplasms induced by low-number pancreatic islet transplants in autoimmune diabetic BB/Pfd rats. Cancer Res 66:1833-1843

37. Bhargava R, Senior PA, Ackerman TE et al (2004) Prevalence of hepatic steatosis after islet transplantation and its relation to graft function. Diabetes 53:1311-1317

38. Markmann JF, Rosen M, Siegelman ES et al (2003) Magnetic resonance-defined periportal steatosis following intraportal islet transplantation: a functional footprint of islet graft survival. Diabetes 52:1591-1594

39. Yasunami Y, Lacy PE, Finke EH (1983) A new site for islet transplantation - a peritoneal-omental pouch. Transplantation 36:181-182

40. Kenyon N, Berman D, O’Neil J et al (2007) Long-term survival of cynomolgus monkey islets implanted in an omental pouch site on tissue engineered scaffolds. Am J Transplant 7 Abstr 1653:572

41. Wells SA Jr, Gunnells JC, Shelburne JD, Schneider AB, Sherwood LM (1975) Transplantation of the parathyroid glands in man: clinical indications and results. Surgery 78:34-44

42. Tominaga Y, Uchida K, Haba T et al (2001) More than 1,000 cases of total parathyroidectomy with forearm autograft for renal hyperparathyroidism. Am J Kidney Dis 38:S168-171

43. Axen KV, Pi-Sunyer FX (1981) Long-term reversal of streptozotocin-induced diabetes in rats by intramuscular islet implantation. Transplantation 31:439-441

44. Dafoe DC, Wang X, Tafra L, Berezniak R, Lloyd RV (1992) Studies of composite grafts of fetal pancreas (FP) and fetal liver $(\mathrm{FL})$ in the streptozotocin-induced diabetic rat. Adv Exp Med Biol 321:171-177

45. Weber CJ, Hardy MA, Pi-Sunyer F, Zimmerman E, Reemtsma K (1978) Tissue culture preservation and intramuscular transplantation of pancreatic islets. Surgery 84:166-174

46. Jovanovic-Peterson L, Williams K, Brennan M, Rashbaum W, Peterson CM (1989) Studies of human fetal pancreatic allografts in diabetic recipients without immunosuppression. J Diabet Complications 3:107-112

47. Tuch BE, Sheil AG, Ng AB, Turtle JR (1986) Long-term survival of human fetal pancreatic tissue transplanted into an insulindependent diabetic patient. Diabet Med 3:24-28

48. Johansson U, Elgue G, Nilsson B, Korsgren O (2005) Composite islet-endothelial cell grafts: a novel approach to counteract innate immunity in islet transplantation. Am J Transplant 5:2632-2639

49. Rafael E, Tibell A, Ryden M et al (2007) Intramuscular autotransplantation of pancreatic islets in a 7-year old child: a two year follow up. Am J Transplant DOI 10.1111/j.1600-6143.2007.02060.x 